\title{
SPRATTUS FUEGENSIS EN AGUAS INTERIORES DE CHILOE, CHILE (OSTEICHTHYES: CLUPEIFORMES: CLUPEIDAE)
}

\author{
SPRATTUS FUEGENSIS IN THE INLAND WATERS OF CHILOE, CHILE \\ (OSTEICHTHYES: CLUPEIFORMES: CLUPEIDAE)
}

\author{
Antonio Aranis R. ${ }^{1}$, Roberto Meléndez C. ${ }^{2}$, Germán Pequeño R. ${ }^{3} \&$ Francisco Cerna T. ${ }^{4}$ \\ ${ }^{1}$ Instituto de Fomento Pesquero, Departamento de Evaluación de Pesquerías, Blanco 839, Valparaíso, Chile. \\ Email: aaranis@ifop.cl. \\ ${ }^{2}$ Museo Nacional de Historia Natural, Casilla 787, Santiago, Chile \\ ${ }^{3}$ Universidad Austral de Chile, Instituto de Zoología, Casilla 567, Valdivia, Chile \\ ${ }^{4}$ Instituto de Fomento Pesquero, Departamento de Especialidades Técnicas, Blanco 839, Valparaíso, Chile.
}

\begin{abstract}
RESUMEN
Una especie de Clupeiformes que ha sido habitual en las capturas de la flota pesquera artesanal que opera en el mar interior de Chiloé, Chile, ha sido confundida con la sardina común (Strangomera bentincki) y la sardina española (Sardinops sagax musica), cuyas distribuciones geográficas en esta área marcan su límite austral. Mediante un análisis de morfometría y recuentos de estructuras duras de siete ejemplares capturados en julio de 2005 y provenientes del área de pesca de Quicaví, Chiloé ( $\left.42^{\circ} 17^{\prime} \mathrm{S}-73^{\circ} 22^{\prime} \mathrm{W}\right)$, se determinó, que correspondían a seis Sprattus fuegensis (Jenyns 1842) (sardina fueguina), y que ella estaría presente hegemónicamente en las capturas del mar interior de Chiloé; en la X Región administrativa de Chile. Basado en la captura de especímenes de $S$. fuegensis obtenidos en las cercanías de la Isla Guar, al norte de Calbuco en octubre de 2005, se realizó una breve descripción de otolitos y una comparación del diámetro longitudinal del primer anillo hialino entre ambas sardinas, mediante el uso del test no paramétrico de Mann-Withney; del mismo modo, se comparó la relación longitud del pez con el diámetro del otolito para ambas sardinas utilizando un ANCOVA. El presente estudio confirma la presencia de S. fuegensis en el océano Pacífico y señala el límite norte de su distribución en el área del Seno de Reloncaví. Este redescubrimiento revela que durante el período de marzo a diciembre de 2005 esta especie sostuvo una actividad extractiva, que viene ocurriendo desde hace algunos años, y que no se ha reflejado oficialmente en las estadísticas de pesca, con desembarques artesanales del orden de las 18 mil toneladas/año. Esto significa que la única pesquería de clupeidos de aguas interiores es sostenida mayoritariamente por S. fuegensis y pone en perspectiva la necesidad de evaluar con cautela los trabajos técnicos y recopilación de información en las aguas interiores de Chiloé.
\end{abstract}

Palabras Claves: Sardina austral, taxonomía, pesquería pelágica.

\section{ABSTRACT}

A specie of Clupeiform which appears in the catch of the artisanal fishing fleet, operating in the inner sea of the southern fjord area of Chiloé, Chile, has been confused with common sardine (Strangomera bentincki) and spanish sardine (Sardinops sagax music) whose geographical distributions in this area delimited its southernmost limit. A morfometric analysis and count of hard structures of six specimens captured on july 2005, coming from the fishing area of Quicaví, Chiloé ( $42^{\circ} 17^{\prime} \mathrm{S}-73^{\circ} 22^{\prime} \mathrm{W}$ ) was determined that as Sprattus fuegensis (Jenyns 1842) (fuegian sprat) and this specie would be the only one present hegemonicaly in the catches of the inland sea of Chiloé, in the X administrative Region of Chile. Based on 33 specimens of S. fuegensis catch near the Isla Guar north of Calbuco in October 2005, a brief description of the otoliths was carried out, also a comparison of the longitudinal diameter of the first hyaline ring, among both sardines using the Mann -Withney non parametric test, in the same way the fish length was compared with the otolith diameter of both sardines, using ANCOVA. The present study confirms the presence of S. fuegensis in southern area of the eastern south Pacific with its northern limit of its distribution in the area of the "Seno de Reloncavî". This rediscovery reveals that during the period from march to december 2005, also S. fuegensis sustained 
an extractive activity for many years ago to date, and has not been reflected officially on the fishery statistics, with artisanal landings about 18,000 ton/year. The fishery of clupeids in inland waters, it is sustained mainly by $S$. fuegensis, which turns out in an exhaustive evaluation, of the technical works and summary of information of inland waters of Chiloé.

KEYwORDs: Southern sardine, taxonomy, pelagic fishery.

\section{INTRODUCCION}

Aunque los peces Clupeiformes han sido ampliamente capturados y desembarcados en la costa de Chile, todavía persisten dificultades en el reconocimiento de los ejemplares, especialmente si se trata de individuos aislados o confinados geográficamente o donde sus distribuciones geográficas se sobreponen. De allí que no sorprende que en la zona sur se denominen como "sardinas" a un conjunto de especies tales como, Engraulis ringens (Jenyns 1842) (anchoveta), juveniles de machuelo o tritre Ethmidium maculatum (Valenciennes 1847), adultos de sardina española, Sardinops sagax (Girard 1854) y sardina común Strangomera bentincki (Norman 1936).

Los primeros estudios taxonómicos sobre clupleiformes, como el catálogo de peces de Delfín (1901), incluye a Clupea fuegensis (Jenyns 1842), recibiendo el nombre vulgar de "sardina común" en la zona de Talcahuano. También especies como Clupea coerulea (Cuvier \& Valenciennes, 1848) para Valparaíso y Clupea arcuata (Jenyns 1841) sensu Gay (1848) in Guichenot, 1948 para los canales de Tierra del Fuego, Canal Beagle y la costa patagónica. Fowler (1945) confirma a Clupea fuegensis presente en localidades similares a las mencionadas por Delfín (1901).

Mann (1954) identifica a Clupea fuegensis (Jenyns 1942) como "sardina común" y señala una distribución desde Valparaíso a Tierra del Fuego. Además, identifica a Clupea bentincki (Norman 1936) como "sardina del sur" o "sardina de invierno" para Talcahuano, y Clupea arcuata (Jenyns 1842) como "sardina de Magallanes" en Magallanes. De Buen (1958) identifica a Clupea (Sprattus) arcuata (Jenyns 1842) para el extremo sur de Chile, a Clupea (Antu) fuegensis (Jenyns 1842) para Tierra del Fuego y Estrecho de Magallanes, Clupea (Antu) bentincki bentincki(Norman 1936) para Talcahuano y Lota; y describe además a Clupea (Antu) bentincki cuga, estableciendo que esta última se distribuye desde Valparaíso hasta Concepción. De Buen
(1959) en su listado de peces chilenos reitera las especies y subespecies indicadas en su trabajo previo.

Posteriormente, Whitehead (1985) en su revisión mundial de sardinas y anchovetas establece que Sprattus fuegensis se distribuiría en el Atlántico sur occidental $\left(40^{\circ} \mathrm{S}\right.$ hasta Tierra del Fuego, incluida las Islas Malvinas), y que los registros de la costa del Pacífico sur oriental se asociarían con el género Strangomera. Así, la especie Strangomera bentincki se distribuiría en el Pacífico sur oriental (Coquimbo, Valparaíso y sur de Talcahuano), y quizás los registros más australes corresponderían a Sprattus fuegensis. Nakamura (1986), señala a Sprattus fuegensis (Jenyns 1842) sólo para la Patagonia argentina y, Clupea bentincki (Norman 1936) para Talcahuano, Chile Central, la Patagonia chilena y además el Estrecho de Magallanes. Pequeño (1989) en su listado sistemático de los peces chilenos, incluye por primera vez como parte de los clupeidos regulares a la especie Sprattus fuegensis (Jenyns 1842). También, Lloris y Rucabado (1991) identifican para el canal Beagle solamente a Sprattus fuegensis. Otros estudios recientes, ignoran su presencia en canales patagónicos chilenos (Madirolas \& Hansen 2000). De acuerdo con Cousseau y Perrotta (1998), los caracteres externos distintivos de Sprattus fuegensis del Atlántico sur occidental serían su cuerpo fusiforme comprimido, la altura comprendida unas cuatro veces y media en el largo total, cabeza relativamente pequeña, ojos de tamaño moderado, boca terminal protráctil estando cerrada y con el extremo posterior llegando a la altura de la mitad del ojo. Mandíbula superior con una muesca amplia en la línea media anterior, ocupada por la mandíbula inferior cuando la boca está cerrada. Mandíbula superior sin dientes, inferior con dientes diminutos en la parte anterior y edéntula en la posterior. Una sola aleta dorsal, cuyo origen es ligeramente anterior a la vertical que pasa por la ventral; la máxima altura corresponde al cuarto o quinto radio. 
Whitehead (1985) señala que los géneros Strangomera y Sprattus pueden ser reconocidos por una diferencia osteológica a nivel del hueso pterótico, que está ubicado en la parte posterior del neurocráneo. En efecto, el género Strangomera presenta una cápsula o bulla timpánica, la cual se encuentra ausente enSprattus. Otro carácter sería el número de radios en la aleta anal, pero este último no es concluyente debido a que la literatura demuestra sobreposición en los rangos.

El tamaño de la sardina fueguina es similar a la sardina común y anchoveta, lo cual lo hace un recurso de talla moderada, y con diferencias poco perceptibles a simple vista, particularmente con la especie S. bentincki. En el litoral argentino, la pesca de esta especie se limita a las capturas costeras y por lo tanto no hay estadísticas oficiales de desembarque. Actualmente no se la explota comercialmente, sin embargo se la ha utilizado para la elaboración de conservas, con buenos resultados (Cousseau \& Perrotta, op cit.).

Debido a la creciente importancia de la pesquería de clupeidos en el mar interior de Chiloé, y la necesidad de disponer de antecedentes biológicos-pesqueros acerca de estos recursos en el área, el año 2005 el Instituto de Fomento Pesquero (IFOP) ejecutó el proyecto FIP 2004-39 "Monitoreo de la pesquería de pequeños pelágicos en aguas interiores de la X Región” (Aranis et al. 2005), en el cual se reconoció la presencia de la especie Sprattus fuegensis en la pesca artesanal de las aguas interiores. En este trabajo se presentan los resultados que permitieron ratificar la presencia de la especie Sprattus fuegensis (sardina fueguina) en los desembarques de aguas interiores y las características relevantes que la diferencian de la especie Strangomera bentincki (sardina común).

\section{MATERIALES Y METODOS}

El área de estudio correspondió a la X Región, la cual latitudinalmente se extiende desde Caleta Queule (39²3'12'S) hasta Punta Guala (4344'17’'S), incluyendo todo el espacio de mar interior, definiéndose cuatro grandes subzonas como son: el Seno de Reloncaví, Golfo de Ancud, Islas interiores de Chiloé y Golfo Corcovado (Figura 1).

La actividad pesquera sobre pelágicos pequeños se desarrolla casi en su totalidad en las tres primeras zonas mencionadas. En el mar interior, sólo puede operar la flota artesanal (naves inferiores o iguales a 50 TRG y 18 metros de eslora), en tanto que la flota industrial está autorizada a operar exclusivamente por fuera de las líneas de base, como es el caso de la flota industrial de cerco, arrastre y mediagua.

Se analizaron inicialmente siete ejemplares de "sardina" proporcionadas por el Instituto de Fomento Pesquero (IFOP) base Calbuco. Estos fueron capturados en la zona de pesca de Quicaví (42 $17^{\circ}$ 'S - 73²2’W, Chiloé) en julio del 2005 por la L/M Pilficán I, cuya descarga fue de 30 toneladas. Además, se dispuso de dos ejemplares identificados como sardina común (Strangomera bentincki) capturados en las cercanías de Puerto Montt, en mayo de 2005. De cada una de las muestras se disectó un ejemplar para ubicar la presencia y/o ausencia de la bulla timpánica, carácter que diferencia el géneroStrangomera de Sprattus (Whitehead 1985). En octubre de 2005 se obtuvo una segunda muestra de 33 ejemplares en las cercanías de la Isla Guar, ligeramente al norte de Calbuco en la costa de Llanquihue (X Región, Chile). Los ejemplares fueron fijados en formalina al $10 \%$ y al cabo de dos semanas fueron cambiados a alcohol etílico $70^{\circ}$. Todos los ejemplares forman parte actualmente de la colección del Museo Nacional de Historia Natural.

Las medidas corporales siguieron lo descrito por Whitehead (1985) y los recuentos de estructuras duras fueron: i) número de radios en las aletas, ii) número de escamas y escudetes, iii) número de branquiespinas en la rama inferior del primer arco branquial, iv) presencia o ausencia de dientes sobre la lengua (Whitehead 1985), v) principales longitudes corporales. Muestras similares fueron sometidas a un análisis morfológico, con el fin de comprobar la pertenencia al orden Clupeiformes y familia Clupeidae. La identificación taxonómica de los especímenes consideró las claves y descripciones propuestas por Fowler (1951), De Buen (1958), Whitehead (1985) y Lloris y Rucabado (1991) (Figura 2 y 3 ).

Se realizó una caracterización de los otolitos encontrados en ejemplares seleccionados de sardina fueguina (9,5 a 18,5 cm longitud total) y de sardina común con el objeto observar probables diferencias entre ambas e identificar posibles mezclas de los stocks en el área de Corral, para ello se comparó el diámetro longitudinal promedio del primer anillo hialino del otolito entre ejemplares de sardina capturados en Talcahuano, Corral y Calbuco, aplicando el test no paramétrico de MannWhitney. Las muestras de Talcahuano y Corral correspondieron a las obtenidas entre julio del 2004 y junio de 2005, en tanto que para la sardina fueguina incluyó muestras de septiembre a noviembre de 2005. 


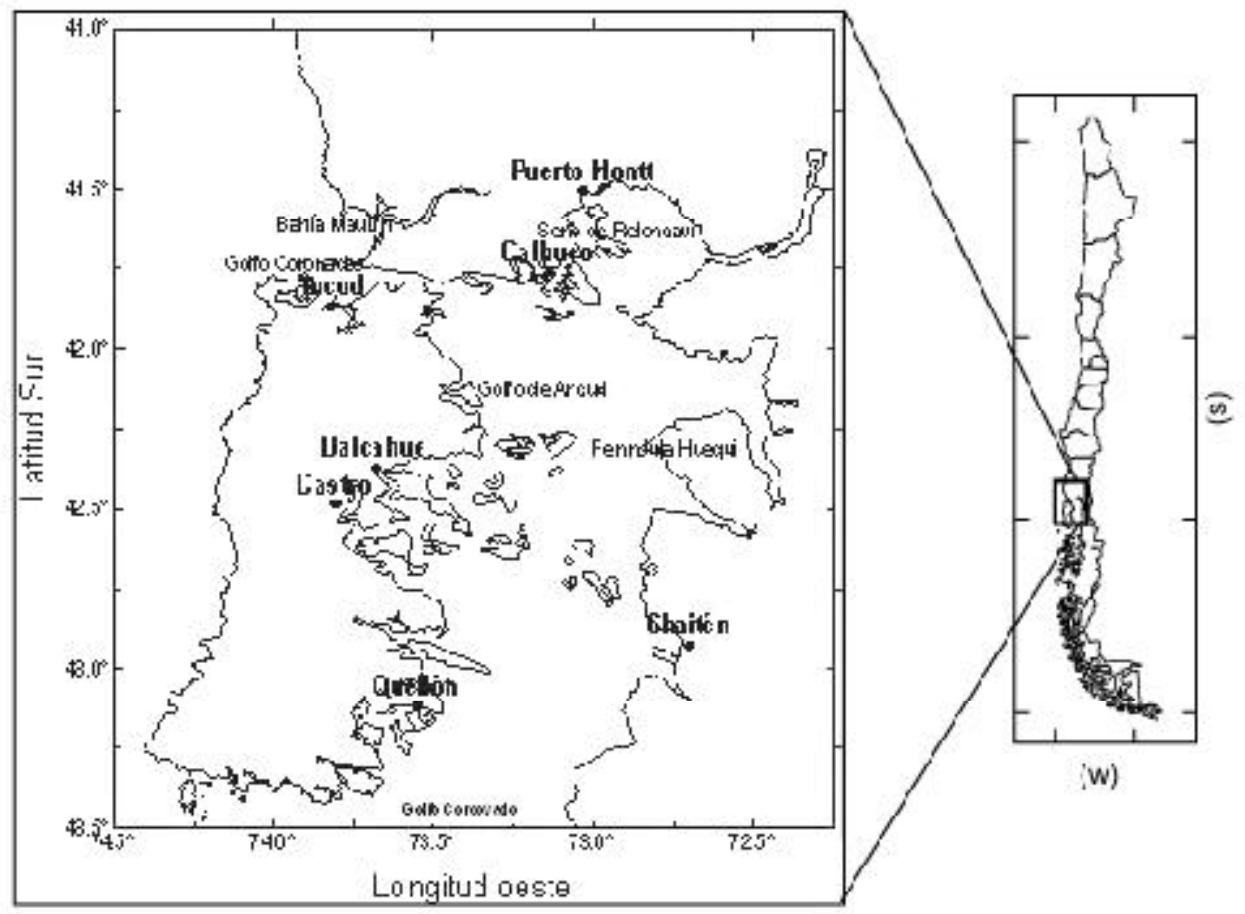

Figura 1. Principales puertos de desembarque de recursos pelágicos en aguas interiores de la X Región.

FIGURE 1. Principal landings ports of pelagic fisheries in the inland waters of Chile X Region.

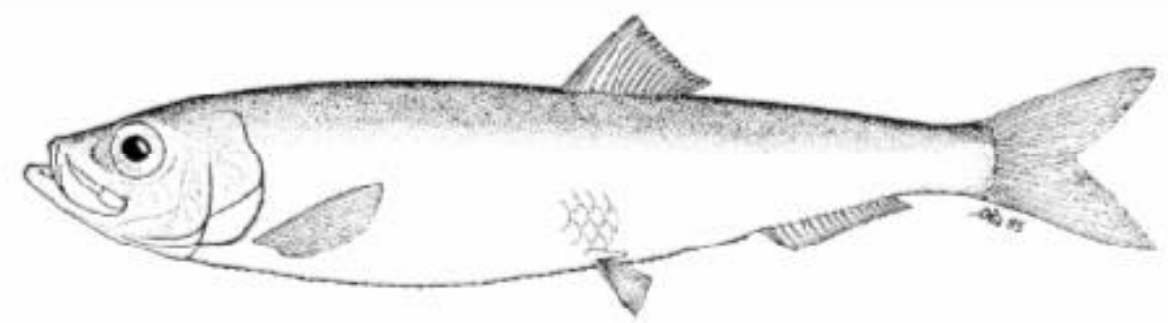

Figura 2. Sprattus fuegensis (Jenyns, 1842), (Whitehead 1985).

Figure 2. Sprattus fuegensis (Jenyns, 1842), (Whitehead 1985). 


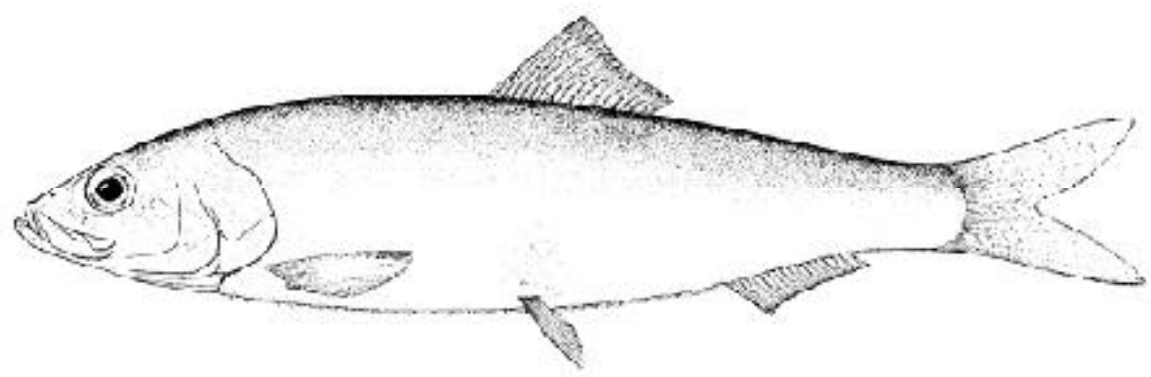

FiguRa 3. Strangomera bentincki (Norman, 1936), (Whitehead 1985).

Figure 3. Strangomera bentincki (Norman, 1936), (Whitehead 1985).

Con el mismo grupo de muestras se estimó la relación longitud del pez con el diámetro del otolito para los ejemplares de sardina capturados en Corral (sardina común) y Calbuco ( sardina fueguina). Se aplicó un análisis de covarianza (ANCOVA) para estimar la existencia de diferencias significativas entre ambas especies, además de determinar si estas relaciones permiten confirmar la distribución descrita de ambas especies en el sur de Chile.

\section{RESULTADOS}

El análisis anatómico en la búsqueda de la bulla timpánica, de un ejemplar identificado "a priori" como Sprattus fuegensis (Figura 2) y de un espécimen de Strangomera bentincki, (Figura 3) reveló que efectivamente ésta estructura osea está ausente en la primera especie y presente en la segunda, confirmando el género de cada una de ellas. En el primer caso el ejemplar corresponde a la muestra colectada en Chile, X Región, Chiloé, Puerto de Calbuco, Quicaví, 13/julio/2005, OYARZO \& ALVARADO (MNHNC P: 7295) y el segundo a la proveniente de Puerto Montt de la colección del Museo Nacional (MNHNC P. sin catalogar).

Los rangos de variación de las medidas corporales y de recuento de estructuras duras de los especimenes estudiados (Tabla I), muestran que tanto la distancia preórbital, distancia postórbital y longitud de la maxila son caracteres útiles para separar especies, en tanto que para el recuento de estructuras sería el número de escudetes de la prealeta pélvica, número de branquiespinas en el arco inferior del primer arco branquial y la presencia de dientes sobre la lengua (Tabla I y Figura 4a y 4b).

En la descripción de $S$. fuegensis del sur Atlántico se confirmó el carácter de no poseer dientes en la mandíbula superior y en la inferior, presentar dientes diminutos en su porción anterior y edéntula en la posterior (Figura 5a y 5b).

De acuerdo a lo anteriormente mencionado, en la primera muestra analizada, seis de los ejemplares obtenidos en Quicaví corresponderían a Sprattus fuegensis, mientras que el ejemplar restante correspondió a Strangomera bentincki.

Los 33 ejemplares recolectados en las cercanías de Calbuco se determinaron como Sprattus fuegensis (Jenyns 1842). Además, se utilizó otros caracteres, como la ausencia de estrías en las piezas operculares (típicas de la sardina común Strangomera bentincki), el cuerpo comparativamente más elongado que en $S$. bentincki. Además, la longitud de la base de la aleta anal es mayor que su análoga la aleta dorsal, característica que es inversa en $S$. bentincki (Tabla I). Elementos claramente diferentes son la posición y abertura de la boca y la ubicación del ojo en el contexto de la cabeza (Figuras 2, 3 y 6). Todos los caracteres anteriores deben ser evaluados con muestras que provengan de todo el rango de distribución geográfica de $S$. fuegensis.

El análisis de los otolitos de los ejemplares de Sprattus fuegensis reflejó la presencia de más de una forma, similar a lo que ocurre en sardina común; sin embargo, a diferencia de esta última, los anillos hialinos se presentan bien marcados y definidos en toda la estructura, lo que facilitó el análisis. 
S. fuegensis en aguas interiores de Chiloé: Aranis, A. ET AL.

TABLA I. Rangos de las mediciones corporales y recuentos para ejemplares de Sprattus fuegensis y Strangomera bentincki provenientes del sur de Chile.

TABLE I. Range of corporal measurements of morphometric and meristic data for Sprattus fuegensis and Strangomera bentincki from southern of Chile.

\begin{tabular}{|c|c|c|c|}
\hline \multirow{3}{*}{ Mediciones corporales (mm) } & \multicolumn{3}{|c|}{ Rangos } \\
\hline & \multirow{2}{*}{$\begin{array}{l}\text { Sprattus fuegensis } \\
\mathrm{n}=6\end{array}$} & \multicolumn{2}{|c|}{ Strangomera bentincki } \\
\hline & & $\mathrm{n}=1$ & $\mathrm{n}=1$ \\
\hline Procedencia & IFOP & IFOP & MUSEO \\
\hline Longitud total & $146-166$ & 136 & 152 \\
\hline \multirow[t]{2}{*}{ Longitud estándar } & $121-140$ & 119 & 125 \\
\hline & $\begin{array}{l}\text { en \% Longitud } \\
\text { estándar }\end{array}$ & $\begin{array}{l}\text { en \% Longitud } \\
\text { estándar }\end{array}$ & $\begin{array}{l}\text { en \% Longitud } \\
\text { estándar }\end{array}$ \\
\hline Longitud cabeza & $25.3-27.3$ & 27.1 & 27.4 \\
\hline Distancia preórbita & $8.0-9.1$ & 7.1 & 6.1 \\
\hline Diámetro órbita & $6.1-7.9$ & 6.6 & 6.2 \\
\hline Distancia postórbita & $10.3-11.7$ & 13.6 & 14.6 \\
\hline Longitud maxila & $11.4-12.3$ & 10.6 & 9.8 \\
\hline Longitud Pre aleta dorsal & $52.2-56.8$ & 52.3 & 51.1 \\
\hline Longitud Pre aleta pélvica & $55.3-57.3$ & 52.4 & 56.6 \\
\hline Longitud Pre aleta pectoral & $24.2-26.8$ & 27.6 & 26.0 \\
\hline Longitud Pre aleta anal & $72.4-76.2$ & 73.8 & 77.1 \\
\hline Base aleta dorsal & $11.3-15.0$ & 14.5 & 15.3 \\
\hline Base aleta anal & $13.4-15.5$ & 13.2 & 13.1 \\
\hline Altura máxima del cuerpo & $22.1-25.3$ & 21.7 & 26.2 \\
\hline Altura mínima pedúnculo caudal & $8.1-9.1$ & 9.1 & 10.1 \\
\hline \multicolumn{4}{|l|}{ Recuentos } \\
\hline$\overline{\text { Aleta dorsal }}$ & $i i+15-i+16$ & $\mathrm{ii}+16$ & $\mathrm{ii}+17$ \\
\hline Aleta anal & $\mathrm{ii}+17-\mathrm{i}+18$ & $\mathrm{ii}+14$ & $\mathrm{ii}+15$ \\
\hline Aleta pélvica & $i+8$ & $i+8$ & $i+9$ \\
\hline Aleta pectoral & $i+16-i+17$ & $i+16$ & $i+17$ \\
\hline Escudetes pre aleta pélvica & $22-23$ & 19 & 19 \\
\hline Escudetes post aleta pélvica & $10-11$ & 11 & 11 \\
\hline Branquiespinas rama inferior & $37-41$ & $<67$ & 76 \\
\hline Presencia dientes sobre la lengua & SI & NO & NO \\
\hline
\end{tabular}

El contorno del otolito presenta crestas, que se observan en mayor proporción en el borde inferior o ventral. En la forma del otolito de las sardinas fueguinas se aprecia que la cauda se presenta unilobulada (roma y aguzada), otra bilobulada que se puede presentar con lóbulo dorsal corto o igual en ejemplares más pequeños, como formas únicas (Figura 7 A a F). En relación al rostro, se encuentran de forma triangular (similar a un triangulo equilátero) y otros con rostro cóncavo en el borde dorsal. La mayoría presenta antirrostro pero en algunos está muy poco definido o ausente.

El diámetro longitudinal del primer anillo hialino en sardina fueguina de Calbuco fue comparado con los valores estimados para sardina común de la zona de Talcahuano y Corral. La sardina fueguina presenta un valor de $1,4 \mathrm{~mm}$ con desviación estándar (DS) 0,2, menor que el registrado en sardina común para la zona de Talcahuano y Corral, que fue de 1,6 mm DS 0,2. El test noparamétrico Mann-Whitney reveló la inexistencia de diferencias significativas ( $\mathrm{p}$ value $=0,731$ ) entre Talcahuano y Corral, lo que confirmaría que serían la misma especie (sardina común), en tanto la comparación estadística entre las sardinas capturadas en Corral y Calbuco mostró diferencias significativas entre los valores promedio de diámetro del primer anillo hialino (p-value=6,05 10$\left.{ }^{46}\right)$. Estos resultados indican que la sardina de Calbuco sería una especie distinta de aquella de Talcahuano y Corral. 
También se estudiaron los parámetros ajustados por una regresión lineal para la relación de crecimiento existente entre la longitud total del pez y el diámetro del otolito de la sardina de Corral (sardina común) y la sardina de Calbuco (sardina fueguina) (Tabla II). Una ANCOVA entre ambas regresiones indica la existencia de diferencias significativas en la relación longitud pez-diámetro del otolito entre las sardinas capturadas en Corral y Calbuco, en pendiente e intercepto ( $\mathrm{p}$ - value $<1,310^{-27}$ ). Esto confirma que en las zonas de captura de Corral y Calbuco (mar interior), efectivamente se presentan especies distintas de sardina con una relación de crecimiento diferente del otolito (Figura 8).
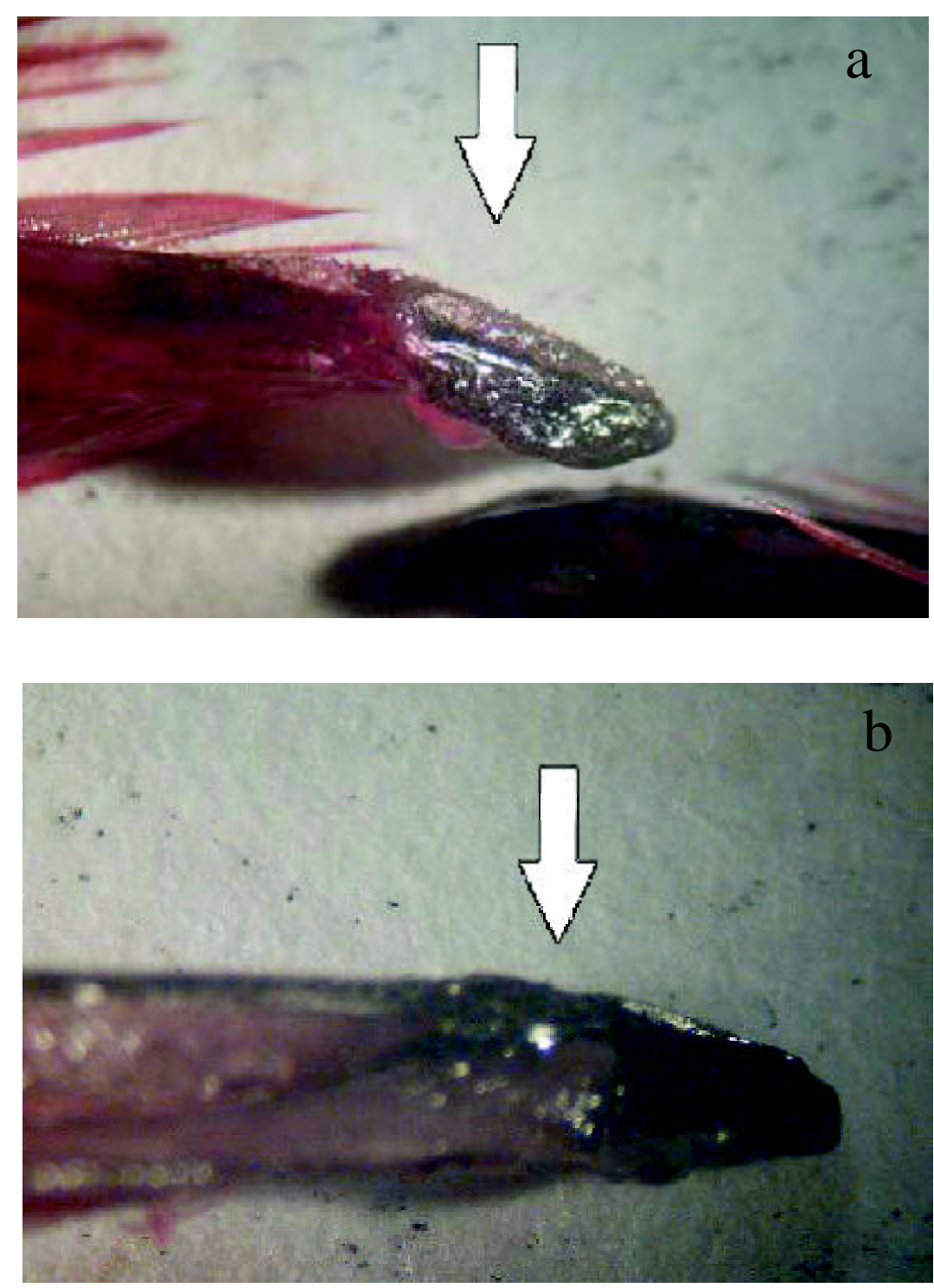

FIgURA 4. Fotografías bajo lupa estereoscópica (10X) de lenguas extirpadas de (a) Sprattus fuegensis y (b) Strangomera bentincki. Las flechas muestran la presencia y ausencia de dientes.

FIGURE 4. Microstereoscopic photograph (10X) of extirped tongues of (a) Sprattus fuegensis and (b) Strangomera bentincki. Arrows show presence and absence of teeth. 


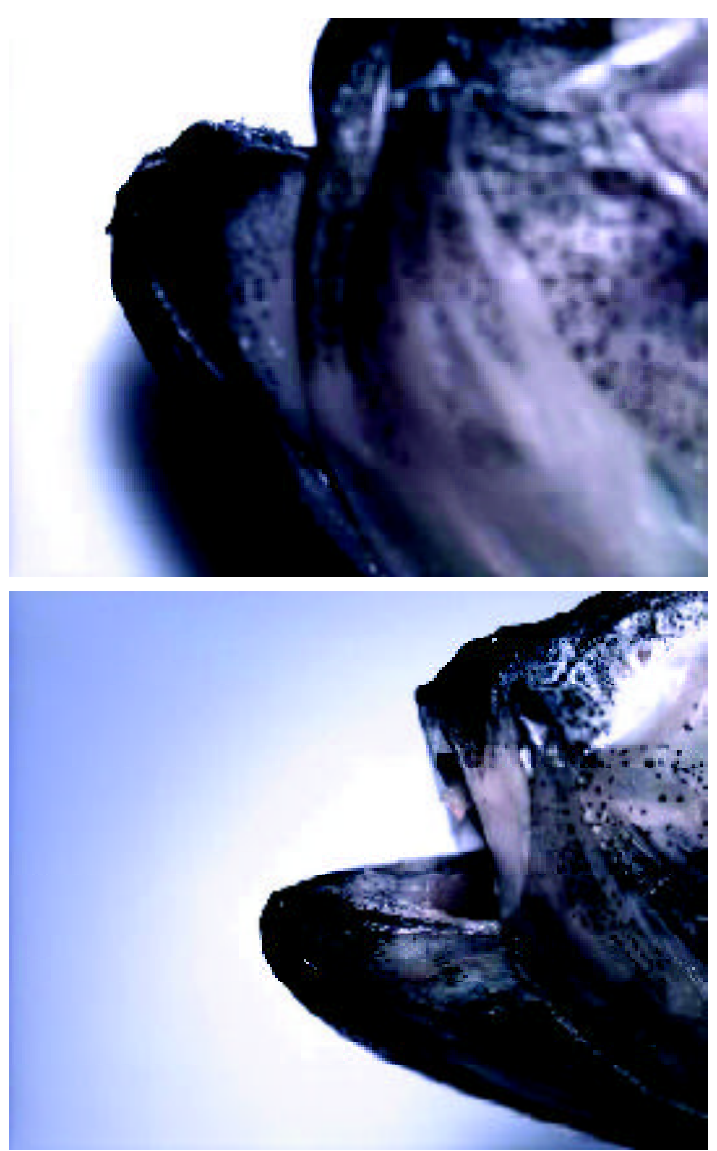

FIGURA 5. Fotografías bajo lupa estereoscópica (10X) de mandíbulas de (a) Sprattus fuegensis y (b) Strangomera bentincki, mostrando la presencia y ausencia de dientes, respectivamente.

FIGURE 5. Microesteroscopic photograph (10X) of jaws of Sprattus fuegensis and (b) Strangomera bentincki. Arrows show presence and absence of teeth respectively.

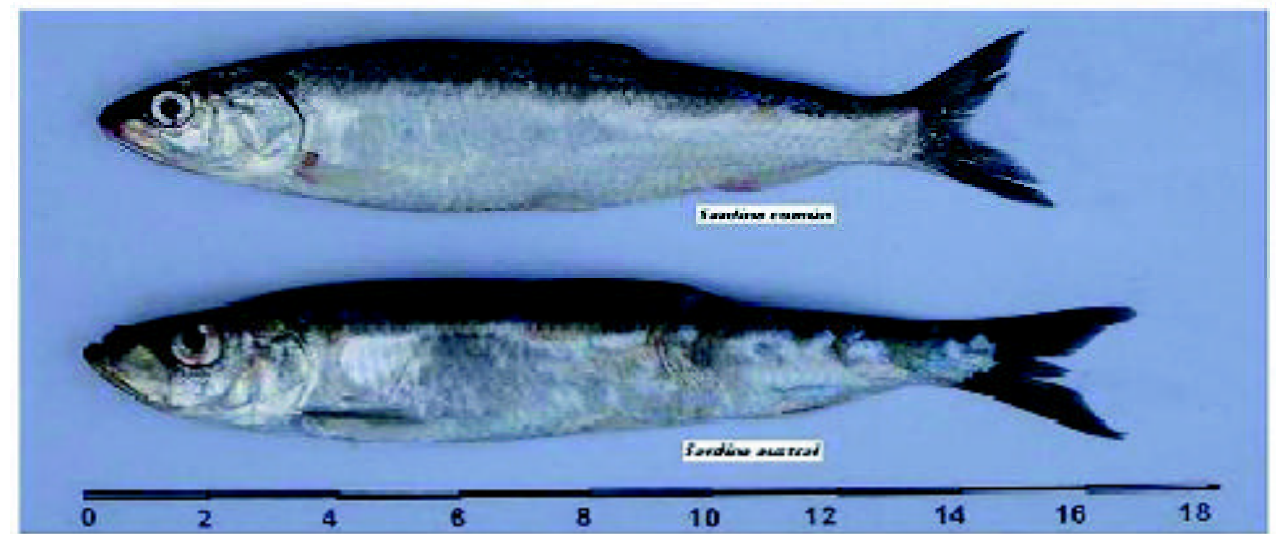

FIGURA 6. Fotografías, (A) sardina común Strangomera bentincki y (B) sardina fueguina Sprattus fuegensis de aguas interiores de la X Región, Chile.

Figure 6. Photographs (A) common sardine Strangomera bentincki and (B) fuegian sprat Sprattus fuegensis, from inland sea of Chilean X Region. 

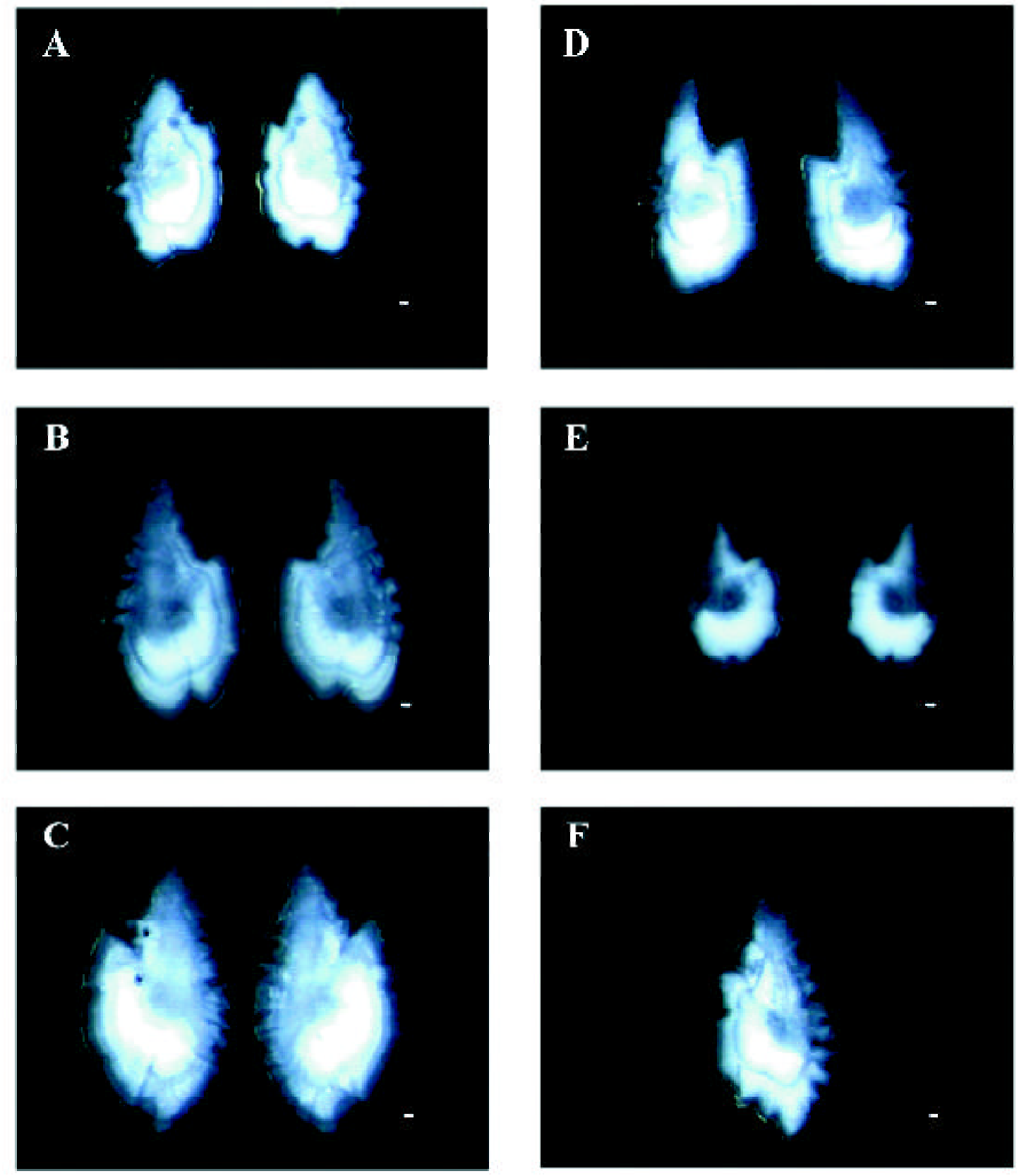

FIgURA 7. Tipos de otolitos sagittae de sardina fueguina (Sprattus fuegensis). Ampliación 30X.

FIGURE 7. Classes of the otoliths sagittae of fuegian sprat (Sprattus fuegensis). Amplification 30X. 


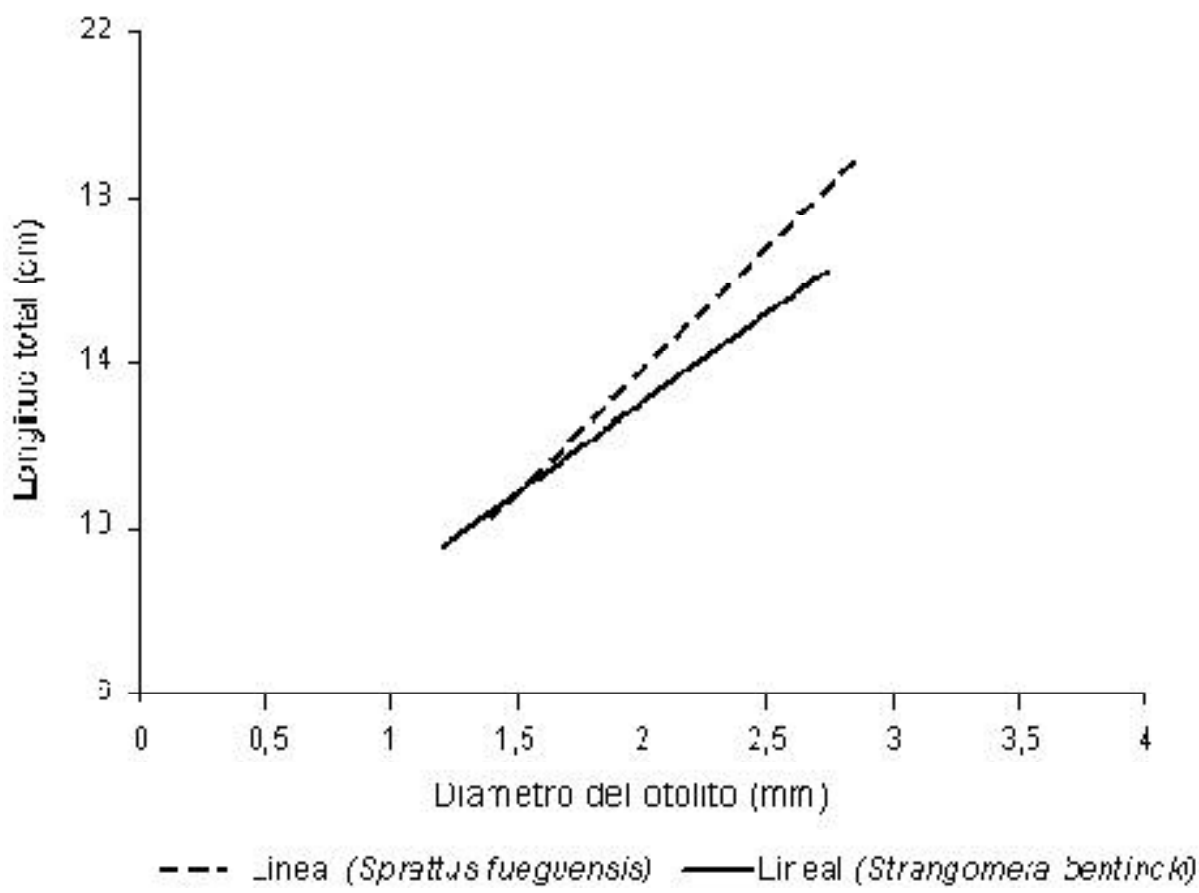

FIGURA 8. Ajuste lineal de la relación entre la longitud del pez y el diámetro del otolito de sardina fueguina (Sprattus fuegensis) de Calbuco (mar interior) y sardina común (Strangomera bentincki) de Corral.

FIGURE 8. Lineal fitting of the relationship between the length of the fish and the diameter of the otoliths of Fuegian sprat (Sprattus fuegensis) of Calbuco (inland sea) and common sardine (Strangomera bentincki) of Corral.

TABLA II. Parámetros de la regresión lineal entre el diámetro del otolito y longitud del pez para sardina común (Corral) y sardina fueguina (Calbuco).

TABLE II. Parameters of the lineal regression between the otolith diameter and longitude of fish for common sardine (Corral) and Fuegian sprat (Calbuco).

\begin{tabular}{ccc}
\hline Parámetros & sardina común & sardina fueguina \\
\hline $\mathrm{a}$ & 4,5 & 2,1 \\
$\mathrm{~b}$ & 4,3 & 5,8 \\
$\mathrm{r} 2$ & 0,72 & 0,89 \\
$\mathrm{n}$ & 1.528 & 688 \\
\hline
\end{tabular}

\section{DISCUSION}

Las características morfológicas analizadas, como la presencia de la bulla timpánica, medidas corporales y recuentos de estructuras permiten concluir que en las zonas de pesca de Calbuco y Quicaví existe la especie Sprattus fuegensis (Jenyns 1842), la cual ha sido confundida por sus similitudes morfológicas con Strangomera bentincki. Aunque la morfología del otolito no es un criterio para la identificación de especie, el análisis de los mismos mostró diferencias evidentes en las características morfológicas, como también en la relación entre diámetro del otolito y la longitud del pez.

Es importante señalar en relación con el reconocimiento taxonómico, que todos los ejemplares estaban en buen estado de preservación, lo que facilitó su medición y la observación reiterada de las características anotadas como diagnósticas. Los antecedentes proporcionados por Norman 1937; Fowler 1951, De Buen 1958, Cousseau 1982, Whitehead 1985 muestran total correspondencia y apoyo a estos resultados. En cuanto a posibles dificultades en el uso de la bibliografía, es necesario mencionar que se observó un error en Fowler $(1942,1943)$, quien cuando trata a Clupea fuegensis, la ilustra, pero sobre la base de una figura que corresponde a Clupea arcuata (Jenyns 1842) y cuando se refiere a Clupea arcuata (Jenyns 1842), la ilustra con una figura de S. fuegensis. 
En cuanto a la forma más sencilla y segura de reconocer las especies involucradas, es la presencia $(S$. fuegensis) y ausencia ( $S$. bentincki) de dientes sobre la lengua. Este carácter no ha sido utilizado por autores previos, probablemente porque no son fácilmente distinguibles a simple vista. Por otra parte, el número de branquispinas en la rama inferior del primer arco branquial es útil para diferenciar las especies en cuestión. Consideramos entonces que menos de 45 corresponde a Sprattus fuegensis y mayor a 65 corresponde a Strangomera bentincki. Cousseau (op. cit.) señala que la sardina común (Clupea bentincki) se distingue de la fueguina, porque tiene branquispinas mucho mas numerosas, indicando que la sardina fueguina presenta 30 a 41 en la rama inferior del primer arco, que es algo menos que los obtenidos en nuestros resultados (37 a 41). En el caso del carácter presencia o ausencia de la bulla timpánica (pterótica), ésta complica su identificación, porque no sólo requiere intervenir el ejemplar sino además es necesario tener un buen conocimiento anatómico y osteológico de peces.

Las diferencias en los porcentajes de algunas medidas corporales y de los recuentos de algunas estructuras duras deben ser sancionadas con un mayor número de muestras. La altura máxima del cuerpo es conveniente explorarla con mayor acuciosidad, ya que en un examen visual, aparentemente $S$. bentincki tendría una mayor altura del cuerpo que S. fuegensis.

Como se señaló, la distribución geográfica de $S$. bentincki y S.fuegensis al sur de Talcahuano no está claramente definida en la literatura, probablemente debido a la escasez de exploraciones y al incipiente desarrollo de las pesquerías en la zona sur, hasta casi fines del siglo XX. La extensión geográfica de estos peces hacia aguas más septentrionales del $\mathrm{Pa}$ cífico sur oriental, solamente ha sido reconocida en años recientes (Zama \& Cárdenas 1984). Pese al hallazgo de Zama \& Cárdenas (op. cit), probablemente poco divulgado, Whitehead (1985) considera que "los registros de la costa del Pacífico (e.g. hasta Valparaíso, Chile, por Mann 1954:130) pueden referirse a Strangomera" y consecuentemente, en el mapa de distribución que presenta para la especie, la limita prácticamente al océano Atlántico sur occidental. Esto, naturalmente ha provocado dudas acerca de la existencia de la especie en Chile. En las aguas de la X Región, se suponía que los principales recursos pelágicos, de acuerdo a las estadís- ticas de pesca, eran sardina común (Strangomera bentincki), anchoveta (Engraulis ringens), jurel (Trachurus murphyi) y pejerrey de mar (Odontesthes regia), los cuales eran obtenidos casi en su totalidad por la flota de cerco artesanal. Dichos recursos se han destinado principalmente a reducción (harina), carnada y consumo humano. Sin embargo, sobre la base de los resultados obtenidos, se confirma la presencia de esta especie hasta el límite norte del litoral del mar interior de la X Región, aproximadamente hasta $\operatorname{los} 41^{\circ} 47,2^{\prime} \mathrm{S}-73^{\circ} 16,5^{\prime} \mathrm{W}$ (sector Aguantao, Ensenada Codihue) a $25 \mathrm{mn}$ al suroeste del Seno de Reloncaví, Puerto Montt, X RegiónChile, lugar más septentrional en que la flota ha realizado lances de pesca exitosos de esta especie. No obstante, es muy probable que el límite natural sea el borde del litoral norte del Seno de Reloncaví. A su vez, la distribución geográfica en la zona patagónica argentina de la sardina fueguina, según Cousseau \& Perrotta (1998), señalan que habita por un lado la plataforma costera patagónica comprendida entre $43^{\circ} 30^{\prime}$ y $55^{\circ} \mathrm{S}$ (costa santacruceña y fueguina) y en latitudes similares, en los alrededores de las Malvinas, lo cual permite establecer un límite circumaustral muy cercano para el lado Atlántico y Pacífico.

Finalmente, se considera de la mayor importancia llevar a cabo estudios sistemáticos y poblacionales sobre sardina común y sardina fueguina en las áreas de mar interior y exterior comprendido entre Valdivia y Punta Arenas.

\section{AGRADECIMIENTOS}

Agradecemos al FIP por las facilidades otorgadas y a los colegas Mariella Canales MSc, Luis Adasme, Alejandra Gómez, Leonardo Caballero y Dr. M. Lamboeuf (FAO) por la reproducción de la figura de Whitehead (1985).

\section{BIBLIOGRAFIA}

Aranis, A., L. Caballero, F. Cerna, A. Gómez, A. López \& C. Bernal. 2005. Informe Pre-Final FIP 200439 "Monitoreo de la Pesquería de Pequeños Pelágicos en Aguas Interiores de la X Región, Año 2004". Fondo de Investigación Pesquera, Inst. Fom. Pesq., Valparaíso, Chile, Chile. 78 p+Anexos.1. 
Cousseau, M.B. 1982. Revisión taxonómica y análisis de los caracteres morfométricos y merísticos de la sardina fueguina, Sprattus fuegensis (Jenyns 1842) (Pisces, Clupeidae). Revista de Investigación y Desarrollo Pesquero, INIDEP, Mar del Plata, 3: 77-94.

Cousseau, M.B. \& R. Perrotta. 1998. Peces Marinos de Argentina, Biología, Distribución, Pesca. Instituto Nacional de Investigación y Desarrollo Pesquero (INIDEP).

De Buen, F. 1958. Peces de la superfamilia Clupoidae en aguas de Chile. Revista de Biología Marina, Valparaíso, 8 (1,2,3):83-110.

De Buen, F. 1959. Lampreas, tiburones, rayas y peces en la Estación de Biología Marina de Montemar, Chile. Revista de Biología Marina, Valparaíso, 9 (1,2,3):3-200.

Delfín, F.T. 1901. Catálogo de los peces de Chile. Revista Chilena de Historia Natural, Imprenta Gillet. Valparaíso, Chile 3-4 (años 1899-1900).

Fowler, H.W. 1942-43. Fishes of Chile, Systematic Catalog, Part I, Revista Chilena de Historia Natural, 46-47: 15-116.

Fowler, H. W. 1951. Analysis of the Fishes of Chile. Revista Chilena de Historia Natural, 51-52: 263-326.

Guichenot, A. 1848. Peces. En: Gay, C. (Ed.) 1848. Historia física y política de Chile. Zoología, v. 2. París \& Santiago.

Lloris, D. \& J. Rucabado. 1991. Ictiofauna del canal Beagle (Tierra del Fuego), aspectos ecológicos y análisis biogeográfico. Publicaciones Especiales Instituto Español de Oceanografía, 8:1-182.

Madirolas, A. \& J.E. Hansen. 2000. Sardina fueguina (Sprattus fuegensis). Pp. 227-232. En: Síntesis del estado de las pesquerías marítimas argenti- nas y de la Cuenca del Plata, años 1997-1998, con una actualización de 1999 (S. Bezzi, R. Akselman y E. Boschi, Eds.). Instituto Nacional de Investigación y Desarrollo Pesquero, Mar del Plata, Argentina, 388 pp.

M ANN, F. G. 1954. Vida de los peces en aguas chilenas. Instituto de Investigaciones Veterinarias del Ministerio de Agricultura y Universidad de Chile, Santiago, 342 pp.

Nakamura, I. 1986. Important fishes trawled off Patagonia. JAMARC. Tokyo, Japan. 369 pp.

Norman, J.R. 1937. Coast fishes, Part II. The Patagonian Region. Discovery Reports, 16: 1-150 + 5 pls.

Pequeño, G. 1989. Peces de Chile. Lista sistemática revisada y comentada. Rev. Biol. Mar., Valparaíso, 24 (2): 1-132.

Shirakova, E.N. 1978a. Some biological features of Tierra del Fuego sprat. Soviet Journal of Marine Biology, 4 (.): 697-702.

Shirakova, E.N. 1978b. Contribution to the biology of Tierra del Fuego spratSprattusfuegensis (Jenyns, 1842). Biologiya Mora, 3: 78-84 (En ruso).

Whitehead, P.J.P. 1985. Clupeoid fishes of the world. An annotated and illustrated catalogue of the herrings, sardines, pilchards, sprats, anchovies and wolfherrings. Part 1. Chirocentridae, Clupeidae and Pristigasteridae. FAO Fisheries Synopsis, 125 (7), part 1: I- x +1-303.

Zama, A. \& E. Cárdenas. 1984. Descriptive catalogue of marine and freshwater fishes from the Aysén region, southern Chile, with zoogeographical notes on the fish fauna. Introduction into Aysén Chile of Pacific salmon. Servicio Nacional de Pesca and Japan International Cooperation Agency, Santiago, 75 pp. + VII Pls. 